Article

\title{
Mesoporous Beta Zeolite Catalysts for Benzylation of Naphthalene: Effect of Pore Structure and Acidity
}

\author{
Wenming Hao $₫$, Weimin Zhang, Zaibin Guo, Jinghong Ma *(i) and Ruifeng Li \\ College of Chemistry and Chemical Engineering, Taiyuan University of Technology, Taiyuan 030024, China; \\ haowenming@tyut.edu.cn (W.H.); zhangweimin_1989@126.com (W.Z.); guozaibin94@163.com (Z.G.); \\ rfli@tyut.edu.cn (R.L.) \\ * Correspondence: majinghong@tyut.edu.cn; Tel: +86-351-6111353
}

Received: 30 September 2018; Accepted: 23 October 2018; Published: 28 October 2018

\begin{abstract}
To improve the catalytic performance of zeolite catalysts in reactions involving bulky molecules, a series of mesoporous Beta zeolite were prepared using organic functionalized fumed silica as the silicon source, which were thoroughly characterized in terms of porosity and acidity. The peaks in X-ray diffraction (XRD) patterns showed broadening, and the external surface area and mesoporosity increased progressively when the content of organic functionalization increased. An infrared (IR) spectroscopy study of adsorbed probe molecules, including pyridine (Py-IR), 2,6-ditertbutylpyridine (DTBPy-IR) and pivalonitrile (Pn-IR), showed that the improvement of mesoporosity increased the accessibility of acidic sites. In the catalytic benzylation of naphthalene with benzyl chloride (BC) over the mesoporous Beta zeolite catalysts, the conversion of BC was significantly increased when the accessibility of Brönsted acid sites improved. The increase of mesoporosity not only improved the diffusion ability of the reactants and products, but also increased the accessibility of acid sites, which greatly enhanced the activity of the mesoporous Beta zeolite catalysts. It is highlighted that the interdependence of mesoporosity, acid type, acid concentration, and strength of the mesoporous Beta zeolites on the catalytic performance in the benzylation of naphthalene with BC was comprehensively studied.
\end{abstract}

Keywords: mesoporous Beta zeolite; accessibility of acid site; mesoporosity; benzylation of naphthalene

\section{Introduction}

Beta zeolite is a microporous crystalline aluminosilicate with a three-dimensional pore system, whose intersecting channels are formed by 12 -membered rings with a diameter of $0.67 \mathrm{~nm}$. Such a zeolite consists of an intergrowth of two structures, i.e., polymorph A and B [1,2]. Beta zeolite with its shape-selective properties and the easy tailoring of their acidity can be synthesized with $\mathrm{Si} / \mathrm{Al}$ ratios between 10 and 100, which is used as a catalyst in a large variety of industrial applications, such as acid-catalyzed hydrocarbon conversions and the selective synthesis of organic compounds [3-6]. However, like many other types of zeolites, the narrow micropores within Beta zeolite could cause slow diffusion of large molecules, which results in a relatively low catalytic activity and a fast catalytic deactivation.

The improvement of zeolite efficiency has been extensively studied, and introducing mesopores into the conventional microporous zeolites is demonstrated to be an effective solution $[7,8]$. Two different strategies have been developed to introduce mesopores into zeolite structures, namely "top-down" (post-synthetic modification) and "bottom-up" (primary syntheses) approaches [9]. The "top-down" approach mostly involved dealumination, desilication, desilication following recrystalization, and surfactant-templated crystal rearrangement; while the "bottom-up" approach involved hard templating, soft templating, assembly of nanosized zeolite, and zeolitization of 
preformed solids [10-13]. Among these approaches, applying organic functionalized silica as the silicon source in the synthesis system of zeolites has been proved to be an effective and practical method making mesoporous zeolites, and the resulted mesoporous zeolites presented enhanced catalytic performance [14-16]. The previous work from our group demonstrated the dramatically improved mesoporosity of a Beta zeolite synthesized by using organic functionalized silica as the silicon source, which showed significantly improved activity in the catalytic reaction for bulky molecules involved in biodiesel production [17].

Friedel-Crafts alkylation is a very important reaction in organic chemistry, which is used in the production of a large variety of chemicals for a wide range of industrial segments. Among these chemicals, benzylated aromatics, which is an important class of alkylated aromatics, are of great importance due to their wide applications in the industrial production of petrochemicals, cosmetics, dyes, pharmaceuticals, and so on. Benzylnaphthalenes are known as useful intermediates for the organic syntheses of additives for lubricants, dyes, and antioxidants, which can be synthesized via the benzylation of naphthalene. Traditional homogeneous acid catalysts for Friedel-Crafts reactions, such as $\mathrm{FeCl}_{3}, \mathrm{AlCl}_{3}, \mathrm{BF}_{3}, \mathrm{ZnCl}_{2}, \mathrm{HF}$, and $\mathrm{H}_{2} \mathrm{SO}_{4}$, are criticized for the high toxicity, corrosion, and difficult recovery and disposal. In contrast, zeolites, known as a kind of solid acid [3], are considered as a green catalyst for the Friedel-Crafts reactions, among which, zeolite H-Beta, H-Y, H-ZSM-5, and H-mordenite were studied in different Friedel-Crafts reactions. However, the microporous zeolites showed poor reactivity in reactions involving large molecules, mainly due to diffusion limitation and low accessibility of the acid sites [18,19]. In comparison, mesoporous zeolite catalysts presented a better performance in large molecules involved in Friedel-Crafts reactions [20-25].

The present work studied the catalytic benzylation of naphthalene with benzyl chloride (BC) over mesoporous Beta zeolite catalysts that were synthesized using organic modified silica as the silicon source. The porosity and acidity were thoroughly characterized and their effect on the catalytic activity in the reaction was investigated comprehensively.

\section{Results and Discussion}

\subsection{Characterization of Mesoporous Beta Zeolites}

Mesoporous Beta zeolite was synthesized by using organic functionalized fumed silica as the silicon source. As shown in the powder X-ray diffraction (XRD) patterns in Figure 1, all the zeolites synthesized from organic functionalized fumed silica exhibited the typical characteristic peaks of a Beta zeolite structure. However, compared with the conventional microporous Beta zeolite (CB) from a non-functionalized silicon source, the mesoporous counterpart (MB1-4) showed broader characteristic peaks when the organic content from the functionalization increased, which could be attributed to the decreased crystalline domains. Such results agree well with our previous findings [15-17].

Porosity of the mesoporous Beta zeolite was characterized using $\mathrm{N}_{2}$ adsorption at $77 \mathrm{~K}$. Figure 2 showed the adsorption and desorption isotherms and the related pore size distributions. In Figure 2a, $\mathrm{CB}$ exhibited an isotherm close to type I according to the IUPAC classification, which was mainly from micropores [26]. Such an isotherm had a sharp increase of adsorption under low $\mathrm{p} / \mathrm{p}_{0}$, and a plateau under higher pressure. While MB1-4 showed different isotherm shapes (Figure 2a), which is a composite of type I and type IV, such isotherms not only have adsorption under low pressure, but also have a continuous increase of adsorption under intermediate and high relative pressures due to the presence of both micropores and mesopores. MB2 exhibited a hysteresis loop in the $\mathrm{p} / \mathrm{p}_{0}$ region between 0.4-1.0. These results are in accordance with our previous findings [15-17]. Accordingly, the pore size distribution from the adsorption branch of the isotherms were calculated using the density functional theory (DFT) model and presented in Figure $2 b$. CB has very limited mesopores according to the pore size distributions. MB1-4 had mesopores in the region between 2-8 nm, among which MB4 showed two main peaks in the mesoporous region: one centered at $3 \mathrm{~nm}$, and the other at $7 \mathrm{~nm}$. 
The pore structure data were also calculated using different models and present in Table 1. The BET surface areas of MB1-4 were higher than that of CB. Moreover, the surface area increased when increasing the content of organic functionalization. CB had a BET surface area of $742 \mathrm{~m}^{2} / \mathrm{g}$, while MB4 had a BET surface area of $931 \mathrm{~m}^{2} / \mathrm{g}$. The increase of BET surface area was cause by the increase of external surface area. Furthermore, the micropore surface area decreased when the mesopore surface area increased. This is because the development of mesopores had to sacrifice micropores, which was also supported by the data of pore volume. When the content of organic functionalization increased, the mesopore volume increased, and the micropore volume decreased accordingly.

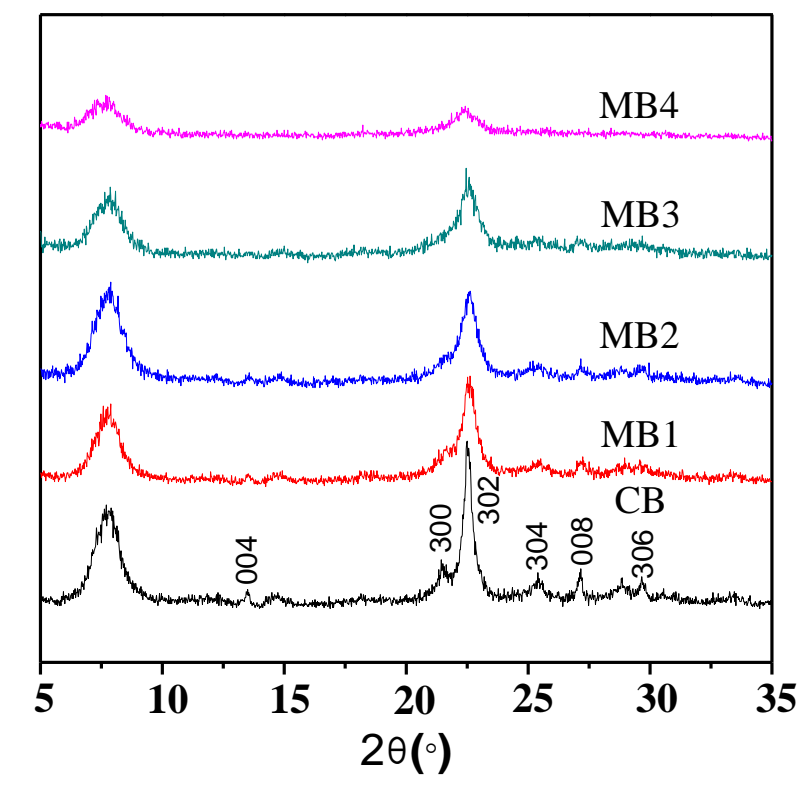

Figure 1. XRD patterns of microporous Beta zeolite CB and mesoporous Beta zeolites MB1-4.
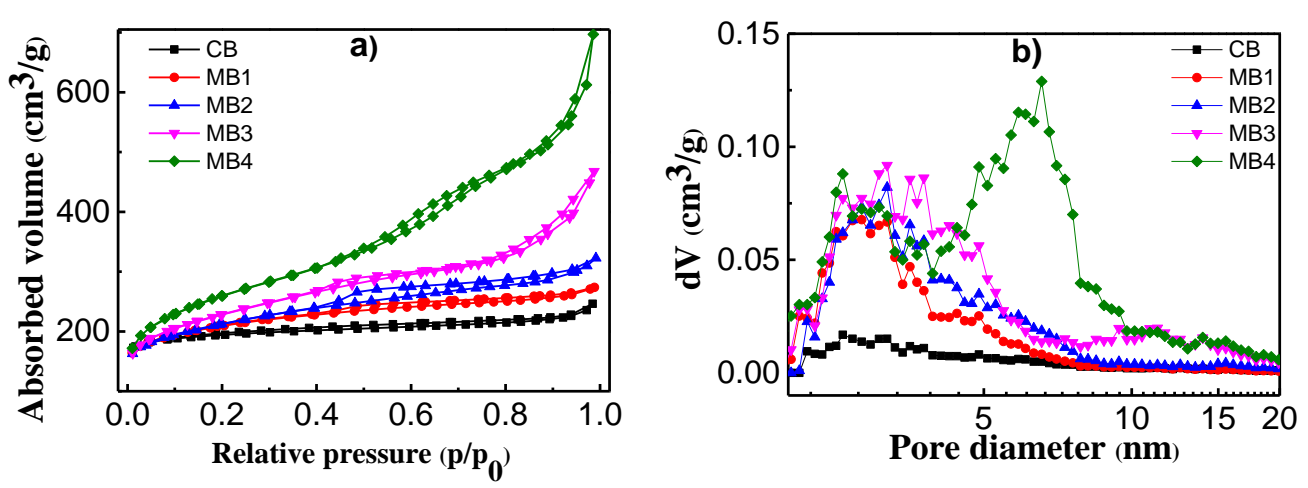

Figure 2. (a) $\mathrm{N}_{2}$ adsorption-desorption isotherms at $77 \mathrm{~K}$ and (b) pore size distribution of microporous Beta zeolite CB and mesoporous Beta zeolite MB1-4.

As described in our previous work, the organic group in the functionalized silicon source hindered the growth of Beta zeolite into large crystals [14,17]. The aggregation of small nanoparticles could form a large amount of mesopores and dramatically increased the external surface area, which was also support by the scanning electron micrograph (SEM) results. Figure 3 compared the SEM images of CB and MB4. Both CB and MB4 had particle sizes of around $500 \mathrm{~nm}$. The particles of CB were constituted of densely stacked sheets with a size of $150 \mathrm{~nm}$. However, the particles of MB4 were aggregated by crystals of smaller than $20 \mathrm{~nm}$. Moreover, in the particles of MB4, the voids between the nanoparticles were bigger than that of $\mathrm{CB}$, which supported the results of increased mesoporosity characterized by $\mathrm{N}_{2}$ adsorption. 


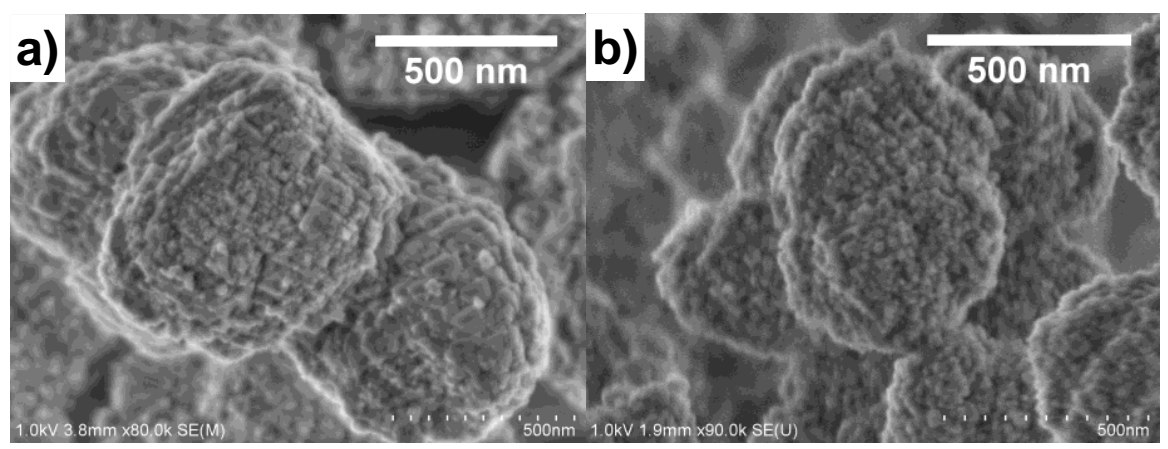

Figure 3. Scanning electron micrographs (SEM) of microporous Beta zeolite CB (a) and mesoporous Beta zeolite MB4 (b).

Table 1. Pore structure properties of microporous Beta zeolite CB and mesoporous Beta zeolite MB1-4.

\begin{tabular}{cccccc}
\hline Sample & $\begin{array}{c}\mathrm{a} \mathbf{S}_{\text {BET }} \\
\left(\mathbf{m}^{2} / \mathbf{g}\right)\end{array}$ & $\begin{array}{c}\mathbf{b} \mathbf{S}_{\text {mic }} \\
\left(\mathbf{m}^{\mathbf{2}} / \mathbf{g}\right)\end{array}$ & $\begin{array}{c}{ }^{\mathrm{c}} \mathbf{S}_{\text {EXT }} \\
\left(\mathbf{m}^{2} / \mathbf{g}\right)\end{array}$ & $\begin{array}{c}\mathbf{d} \mathbf{V}_{\text {mic }} \\
\left(\mathbf{c m}^{3} / \mathbf{g}\right)\end{array}$ & $\begin{array}{c}\mathbf{e} \mathbf{V}_{\text {meso }} \\
\left(\mathbf{c m}^{3} / \mathbf{g}\right)\end{array}$ \\
\hline CB & 742 & 679 & 63 & 0.28 & 0.05 \\
MB1 & 764 & 614 & 150 & 0.27 & 0.15 \\
MB2 & 764 & 573 & 191 & 0.26 & 0.24 \\
MB3 & 814 & 472 & 342 & 0.22 & 0.50 \\
MB4 & 931 & 375 & 556 & 0.16 & 0.92 \\
\hline
\end{tabular}

${ }^{a} S_{\text {BET }}$ is the BET surface area obtained from the $\mathrm{N}_{2}$ adsorption isotherm; ${ }^{b} \mathrm{~S}_{\text {mic }}$ is the micropore surface area; ${ }^{c} \mathrm{~S}_{\mathrm{EXT}}$ is the external surface area; ${ }^{d} V_{\text {mic }}$ is the micropore volume; ${ }^{e} V_{\text {meso }}$ is the mesopore volume $\left(V_{\text {total }}-V_{\text {mic }}\right)$; $S_{\text {mic }}$, $\mathrm{S}_{\mathrm{EXT}}$, and $\mathrm{V}_{\text {mic }}$ were obtained from the T-plot method.

The chemical environment on the surface of the zeolite was different from that inside the crystal. Figure 4 showed the $\mathrm{OH}$ region of infrared (IR) spectra for $\mathrm{CB}$ and $\mathrm{MB} 4$, which have different external surface areas and different $\mathrm{OH}$ groups. The peak at $3745 \mathrm{~cm}^{-1}$ was assigned to external silanol groups, and the peak at $3735 \mathrm{~cm}^{-1}$ could be attributed to internal silanol groups [27]. MB4 showed a high intensity of the external surface silanol group peak at $3745 \mathrm{~cm}^{-1}$, which was ascribed to the increased external surface area. On the contrary, CB showed a high intensity of the internal silanol peak at $3735 \mathrm{~cm}^{-1}$, which was in accordance with the fact that the micropore was dominant in CB.

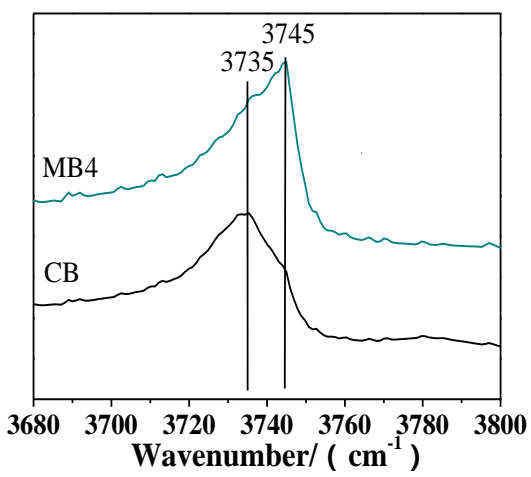

Figure 4. Partial enlargement of hydroxyl infrared (IR) spectra of microporous Beta zeolite CB and mesoporous Beta zeolite MB4.

The difference in the chemical environment for $\mathrm{CB}$ and MB1-4 were characterized by ${ }^{29} \mathrm{Si}$ MAS NMR as well. Figure 5 presented the ${ }^{29} \mathrm{Si}$ MAS NMR spectra of CB and MB4 $\left({ }^{29} \mathrm{Si}\right.$ MAS NMR spectra of MB1-3 are shown in Figure S1). Five peaks assigned to $\mathrm{Q}^{4}(-115$ and $-111 \mathrm{ppm}), \mathrm{Q}^{3}(-107$ and $-103 \mathrm{ppm})$, and $\mathrm{Q}^{2}(-95 \mathrm{ppm})$ sites were detected when using Gaussian functions fitting the peaks. Two peaks for $\mathrm{Q}^{4}$ sites originated from the two different stacking orders of polymorph $\mathrm{A}$ 
and polymorph $\mathrm{B}$, which are typical for zeolite BEA. The $\mathrm{Q}^{3}$ peaks at -107 and $-103 \mathrm{ppm}$ were from $\mathrm{Si}(\mathrm{OSi})_{3}(\mathrm{OAl})_{1}$ and $\mathrm{Si}(\mathrm{OSi})_{3}(\mathrm{OH})_{1}$ tetrahedrons, respectively [28]. The $\mathrm{Q}^{2}$ peak at $-95 \mathrm{ppm}$ was assigned to the $\mathrm{Si}(\mathrm{OSi})_{2}(\mathrm{OAl})_{2}$ tetrahedron. It was noticed that the peaks of $\mathrm{MB} 4$ were wider than that of $\mathrm{CB}$, which demonstrated that the mesoporous Beta zeolite from organic functionalized silicon showed a decreased homogeneous chemical environment compared with that of the microporous Beta zeolite. Such changes are largely attributed to the increased external surface area of the mesoporous Beta zeolite.
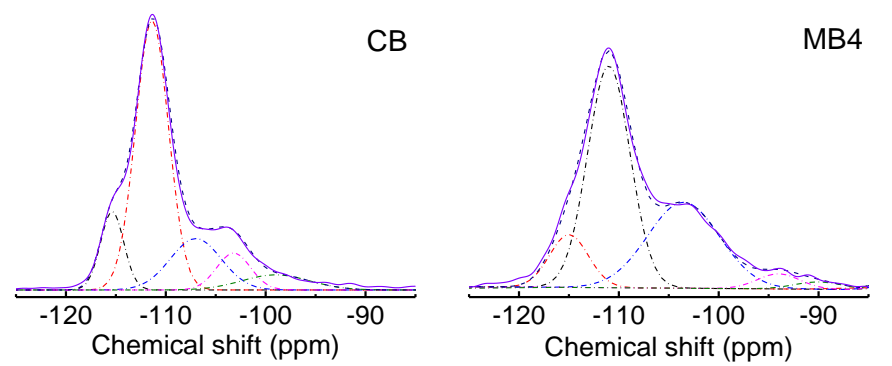

Figure 5. ${ }^{29}$ Si MAS NMR spectra of microporous Beta zeolite CB and mesoporous Beta zeolite MB4. Solid line is the original data, the dash-dot line is the fitted data by Gaussian functions, and the dashed line is the cumulative fitted data.

\subsection{Acidity of Mesoporous Beta Zeolites}

There are two families of techniques, namely spectrometric (IR and NMR) and adsorption-desorption methods (calorimetry and temperature programed desorption (TPD)), that are normally used to characterize the acidity of zeolites [29,30]. In this study, bulk acidity and mesopore surface acidity was investigated by using temperature-programmed desorption of ammonia $\left(\mathrm{NH}_{3}-\mathrm{TPD}\right)$ and infrared (IR) spectroscopic studies of adsorbed probe molecules with different sizes (pyridine (Py), 2,6-di-tert-butylpyridine (DTBPy), and pivalonitrile (Pn)).

\subsubsection{Bulk Acidity of Mesoporous Beta Zeolites by $\mathrm{NH}_{3}$-TPD}

$\mathrm{NH}_{3}$-TPD was applied to characterize the bulk acidity of mesoporous Beta zeolites, including acid strength and the relative amount of acid with different strengths. The temperature of desorption is related to the strength of the acid. Strong acid sites have a more stable interaction with $\mathrm{NH}_{3}$ molecules, which need a higher temperature to be desorbed. Figure $\mathrm{S} 2$ compared the $\mathrm{NH}_{3}-\mathrm{TPD}$ data of $\mathrm{CB}$ and MB1-4. The desorption of $\mathrm{NH}_{3}$ could be divided into contributions from weak (desorption maximum at $490-504 \mathrm{~K}, \mathrm{~T}_{\mathrm{L}}$ ), medium (desorption maximum at 526-556 K, $\mathrm{T}_{\mathrm{M}}$ ), and strong (desorption maximum at $631-649 \mathrm{~K}, \mathrm{~T}_{\mathrm{H}}$ ) acid sites. The low-temperature peak was assigned to the desorption of $\mathrm{NH}_{3}$ from Brönsted acid sites. The strong acid sites are related to Lewis acid sites that bind strongly to $\mathrm{NH}_{3}$ and need a high temperature for desorption [28]. The calculated results are summarized in Table 2. It can be noticed that the mesoporous Beta zeolites MB1-4 did not present a big difference in the acid strength from the microporous Beta zeolite CB.

Table 2. $\mathrm{NH}_{3}-\mathrm{TPD}$ data of microporous Beta zeolite $\mathrm{CB}$ and mesoporous Beta zeolites MB1-4.

\begin{tabular}{cccccccc}
\hline Sample & $\mathbf{S}_{\text {EXT }}\left(\mathbf{c m}^{\mathbf{2}} \mathbf{g}\right)$ & ${ }^{\mathbf{a}} \mathbf{T}_{\mathbf{L}}(\mathbf{K})$ & ${ }^{\mathbf{b}} \mathbf{T}_{\mathbf{M}}(\mathbf{K})$ & ${ }^{\mathbf{c}} \mathbf{T}_{\mathbf{H}} \mathbf{( K )}$ & ${ }^{\mathbf{d}} \mathbf{A}_{\mathbf{T L}} \mathbf{( \% )}$ & ${ }^{\mathbf{e}} \mathbf{A}_{\mathbf{T M}} \mathbf{( \% )}$ & ${ }^{\mathbf{f}} \mathbf{A}_{\mathbf{T H}} \mathbf{( \% )}$ \\
\hline CB & 63 & 508 & 542 & 640 & 11.7 & 17.3 & 71.0 \\
MB1 & 150 & 497 & 536 & 634 & 13.7 & 21.0 & 65.3 \\
MB2 & 191 & 491 & 530 & 629 & 12.4 & 19.6 & 68.0 \\
MB3 & 342 & 489 & 525 & 629 & 11.8 & 22.0 & 66.2 \\
MB4 & 556 & 504 & 556 & 649 & 21.0 & 29.5 & 49.5 \\
\hline
\end{tabular}

${ }^{a} \mathrm{~T}_{\mathrm{L}}$ is the low temperature of the desorption of $\mathrm{NH}_{3} ;{ }^{\mathrm{b}} \mathrm{T}_{\mathrm{M}}$ is the medium temperature of the desorption of $\mathrm{NH}_{3}$;

${ }^{\mathrm{c}} \mathrm{T}_{\mathrm{H}}$ is the high temperature of the desorption of $\mathrm{NH}_{3} ;{ }^{\mathrm{d}} \mathrm{A}_{\mathrm{TL}}$ is the relative concentration of acid sites determined at $\mathrm{T}_{\mathrm{L}} ;{ }^{\mathrm{e}} \mathrm{A}_{\mathrm{TM}}$ is the relative concentration of acid sites determined at $\mathrm{T}_{\mathrm{M}} ;{ }^{\mathrm{f}} \mathrm{A}_{\mathrm{TH}}$ is the relative concentration of acid sites determined at $\mathrm{T}_{\mathrm{H}}$. 


\subsubsection{Bulk Acidity of Mesoporous Beta Zeolites Using Py-IR}

Pyridine (Py) is a basic molecule with a kinetic molecular size of $0.54 \mathrm{~nm}$, which is considered to be capable of being adsorbed on the Brönsted acid sites and Lewis acid sites within both the micropores and on the external surface of Beta zeolite [31]. Py-IR is a widely used method to characterize the total amount of acid in a porous material. As shown in Figure 6 (MB4) and Figure S3 (CB and MB1-3), the IR band at $1546 \mathrm{~cm}^{-1}$ was assigned to Py adsorbed on Brönsted acid sites, the band at $1456 \mathrm{~cm}^{-1}$ was attributed to Py adsorbed on Lewis acid sites, and the band at $1490 \mathrm{~cm}^{-1}$ was from Py adsorbed on both Brönsted acid and Lewis acid sites [32]. The band at $1456 \mathrm{~cm}^{-1}$ had a shoulder at $1445 \mathrm{~cm}^{-1}$ which was related to Py adsorbed on a Si-OH group. It can be noticed that the intensity of all the bands decreased when adsorption temperature increased, which was due to the desorption of the adsorbed Py from weak acid sites. The Py remaining at higher temperatures were those adsorbed on strong acid sites. Interestingly, the shoulder band at $1445 \mathrm{~cm}^{-1}$ disappeared when the temperature increased from $423 \mathrm{~K}$ to $623 \mathrm{~K}$, which was related to the desorption of Py from a Si-OH group at higher temperatures. The concentration of both Brönsted acid and Lewis acid sites were calculated from the Py-IR spectra and tabulated in Table 3. The concentration of both Brönsted acid and Lewis acid sites decreased when the temperature increased because of the desorption of Py at high temperatures.

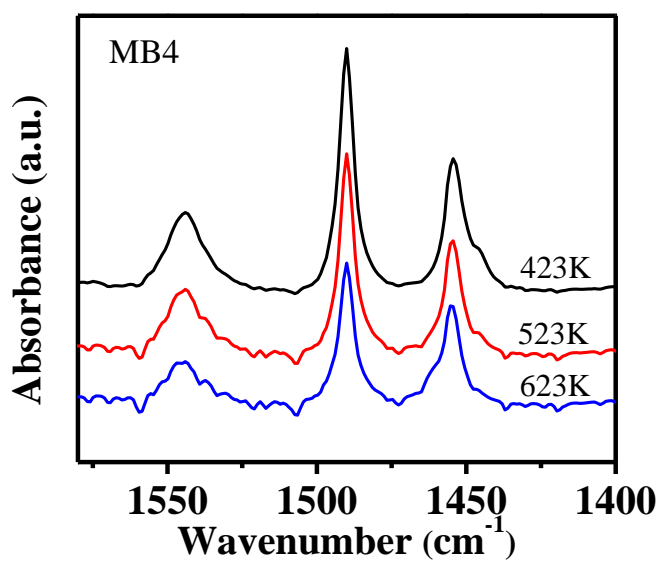

Figure 6. Py-IR spectra of mesoporous zeolite MB4 after the desorption of pyridine (Py) at three different temperatures.

Table 3. Infrared (IR) spectra data of microporous Beta zeolite CB and mesoporous Beta zeolites (MB1-4) adsorbed with different probe molecules.

\begin{tabular}{|c|c|c|c|c|c|}
\hline \multirow{2}{*}{ Sample } & \multicolumn{3}{|c|}{ Py-IR } & \multirow{2}{*}{$\begin{array}{c}\text { DTBPy-IR } \\
\begin{array}{c}\mathrm{B}_{\mathrm{DTBPy}} \\
(\mu \mathrm{mol} / \mathrm{g}, 423 \mathrm{~K})\end{array}\end{array}$} & \multirow{2}{*}{$\begin{array}{c}\text { Pn-IR } \\
L_{P n} \\
(\mu \mathrm{mol} / \mathrm{g}, 303 \mathrm{~K})\end{array}$} \\
\hline & $\mathrm{L}_{\mathrm{Py}}(\mu \mathrm{mol} / \mathrm{g})$ & $\mathrm{B}_{\mathrm{Py}}(\mu \mathrm{mol} / \mathrm{g})$ & $\begin{array}{c}\text { Py-B + L } \\
(\mu \mathrm{mol} / \mathrm{g}, 423 \mathrm{~K})\end{array}$ & & \\
\hline \multirow{3}{*}{$\mathrm{CB}$} & $101(423 \mathrm{~K})$ & 209 (423 K) & \multirow{3}{*}{310} & \multirow{3}{*}{-} & \multirow{3}{*}{-} \\
\hline & $71(523 \mathrm{~K})$ & 206 (523 K) & & & \\
\hline & $60(623 \mathrm{~K})$ & 179 (623 K) & & & \\
\hline \multirow{3}{*}{ MB1 } & $189(423 \mathrm{~K})$ & 177 (423 K) & \multirow{3}{*}{366} & \multirow{3}{*}{37} & \multirow{3}{*}{117} \\
\hline & $144(523 \mathrm{~K})$ & $162(523 \mathrm{~K})$ & & & \\
\hline & $112(623 \mathrm{~K})$ & $96(623 \mathrm{~K})$ & & & \\
\hline \multirow{3}{*}{ MB2 } & $168(423 \mathrm{~K})$ & $208(423 \mathrm{~K})$ & \multirow{3}{*}{376} & \multirow{3}{*}{81} & \multirow{3}{*}{118} \\
\hline & 145 (523 K) & 194 (523 K) & & & \\
\hline & $111(623 \mathrm{~K})$ & $115(623 \mathrm{~K})$ & & & \\
\hline \multirow{3}{*}{ MB3 } & $112(423 \mathrm{~K})$ & $152(423 \mathrm{~K})$ & \multirow{3}{*}{264} & \multirow{3}{*}{92} & \multirow{3}{*}{120} \\
\hline & $98(523 \mathrm{~K})$ & $131(523 \mathrm{~K})$ & & & \\
\hline & $79(623 \mathrm{~K})$ & $92(623 \mathrm{~K})$ & & & \\
\hline \multirow{3}{*}{ MB4 } & $181(423 \mathrm{~K})$ & $241(423 \mathrm{~K})$ & \multirow{3}{*}{422} & \multirow{3}{*}{216} & \multirow{3}{*}{164} \\
\hline & $144(523 \mathrm{~K})$ & $198(523 \mathrm{~K})$ & & & \\
\hline & $132(623 \mathrm{~K})$ & $123(623 \mathrm{~K})$ & & & \\
\hline
\end{tabular}




\subsubsection{Accessibility of Acids over Mesoporous Beta Zeolites by DTBPy-IR}

Another basic probe molecule 2,6-di-tert-butylpyridine (DTBPy) has a kinetic molecular size of $0.79 \mathrm{~nm}$, which is considered to be unable to entirely enter the micropores of Beta zeolite. Because of that, DTBPy-IR is often used to detect the accessible Brönsted acid sites of zeolite crystals [33]. Figure S4 presents the DTBPy-IR spectra of mesoporous Beta zeolites. The bands at $1613 \mathrm{~cm}^{-1}$ were related to the DTBPy molecule adsorbed in mesoporous Beta zeolites. The concentration of the Brönsted acid sites were calculated from the area of the bands and are shown in Table 3. It can be noticed that the concentration of Brönsted acid sites increased from $32 \mu \mathrm{mol} / \mathrm{g}$ for MB1 to $216 \mu \mathrm{mol} / \mathrm{g}$ for MB4. This was because the increase of external surface area meant more acid sites were exposed to the large molecule. MB4 had a similar concentration of Brönsted acid sites from DTBPy-IR $(216 \mu \mathrm{mol} / \mathrm{g})$ when compared the data from Py-IR $(241 \mu \mathrm{mol} / \mathrm{g})$, which meant the majority of the Brönsted acid sites were exposed on the external surface of the zeolite. Such acid sites are of great importance to the catalytic performance of the zeolite catalyst in reactions involving bulky molecules.

\subsubsection{Accessibility of Acids over Mesoporous Beta Zeolites by Pn-IR}

DTBPy-IR is widely used to determine the concentration of Brönsted acid sites on the external surface of zeolites. However, the drawback for DTBPy-IR is that Lewis acid sites cannot be detected by such a method. IR spectrometry of adsorbed pivalonitrile (Pn) with large molecular size was developed to study the accessible Lewis acid sites on the surface of zeolites [34]. In this study, Pn-IR was used as a supplementary method to study the concentration of Lewis acid sites on the external surface of mesoporous Beta zeolite. Figure S5 shows a different shape of the Pn-IR spectra of the mesoporous Beta zeolites. Gaussian functions were used to fit the peaks. The band at $2294 \mathrm{~cm}^{-1}$ was assigned to Pn molecules adsorbed by Lewis acid sites and the band at $2277 \mathrm{~cm}^{-1}$ was related to Pn molecules adsorbed by Brönsted acid sites. The concentration of Lewis acid sites on the external surface was calculated from the Gaussian functions fitted peaks and is listed in Table 3. As can be seen in Table 3, the concentration of Lewis acid sites from Pn-IR of the studied mesoporous Beta zeolites increased slowly, which was due to the increase of the external surface area, and the majority of the Lewis acid sites were located on the external surface. It has been widely accepted that Lewis acid sites are related to the extra-framework $\mathrm{Al}$ [35]. In mesoporous Beta zeolite, the increase of external surface area will generate lots of extra-framework $\mathrm{Al}$, which results in more Lewis acid sites.

\subsection{Benzylation of Naphthalene over Mesoporous Beta Zeolite}

Figure 7 is the illustration of the catalytic benzylation of naphthalene with benzyl chloride (BC) conducted over mesoporous Beta zeolite catalyst. Naphthalene has a kinetic molecular size of $0.50 \mathrm{~nm} \times 0.68 \mathrm{~nm}$, which is very close to the pore size of Beta zeolite; the product benzylnaphthalenes $(\mathrm{BN})$ had kinetic molecular sizes around $1.0 \mathrm{~nm}$, which is not possible to form within the micropore of Beta zeolite. Only the active sites on the pore mouth are able to be accessed by such large molecules. Two different isomers were detected in the products ( $\alpha-\mathrm{BN}$ and $\beta-\mathrm{BN})$, and are tabulated in Table S1. The selectivity of $\alpha$-BN was around $80 \%$, much higher than that of $\beta$-BN for all the studied catalysts, which was due to the smaller diameter of $\alpha$-BN being easier to diffuse in the pathways of the catalysts. The mesoporous Beta zeolites (MB1-4) did not show much difference from the microporous Beta zeolite CB in terms of their selectivity.

As shown in Figure 8a, the conversion of $\mathrm{BC}$ increased when the external surface area of the mesoporous Beta zeolite catalysts increased. After $8 \mathrm{~h}$ of reaction, the conversion of BC reached $100 \%$ over MB4, with an external surface area of $556 \mathrm{~cm}^{2} / \mathrm{g}$. The catalyst recycling in the model reactions had been experimentalized in our laboratory. After the catalysts were used three times, the catalytic results had no apparent changes, and the XRD patterns of the used catalysts had no noticeable changes compared with the fresh catalysts. The low conversion of BC over microporous Beta zeolite (CB) catalysts with a low external surface area could be ascribed to that of the acidic 
sites within the micropores of Beta zeolites, which were not accessible to large reactant molecules. Those acidic sites played a crucial role as the active sites. Interestingly, the conversion of BC increased gradually when the external surface area increased. The mesopores with a large external surface area could improve the diffusion, prohibit it from the blockage of acidic sites, and improve the accessibility of acidic sites. It is worth noticing in Figure 8a that the accessibility of Brönsted acid sites increased with the improvement of the external surface area, which contributed to the high conversion of BC. The accessibility of acid sites could benefit the catalytic performance of the Beta zeolite catalyst, since it helped to form carbenium ions from large molecules during the reaction [6,27].

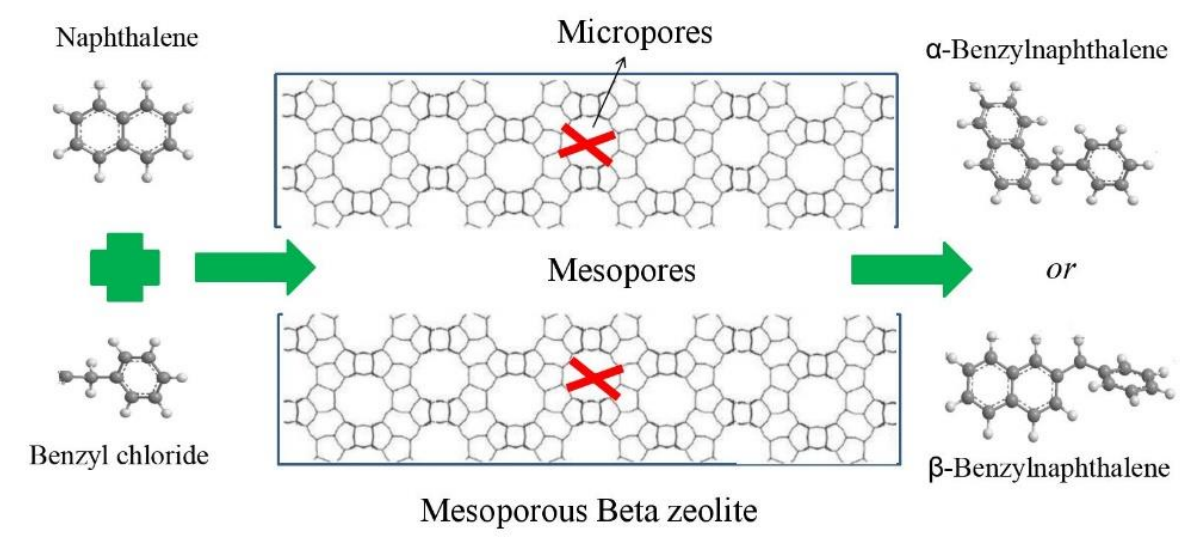

Figure 7. Illustration of the catalytic reaction of the benzylation of naphthalene with benzyl chloride (BC) over mesoporous Beta zeolite.
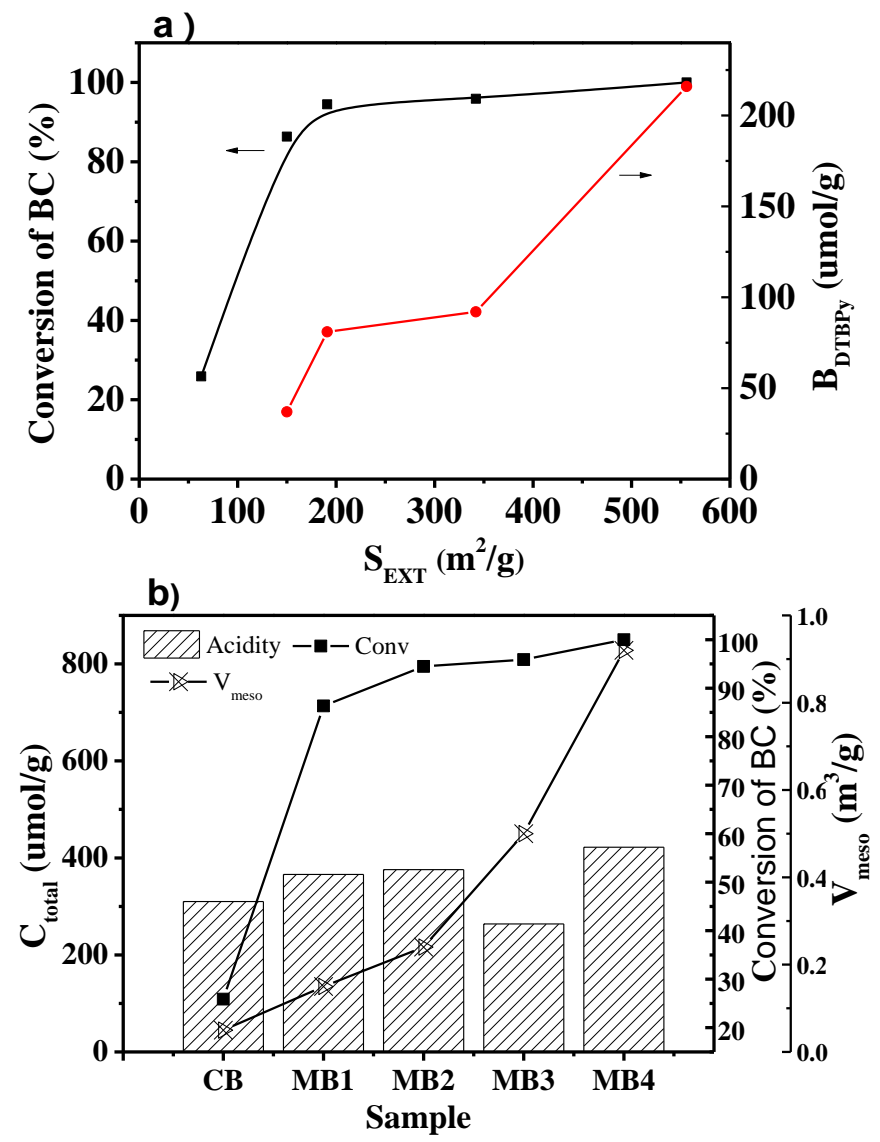

Figure 8. Cont. 


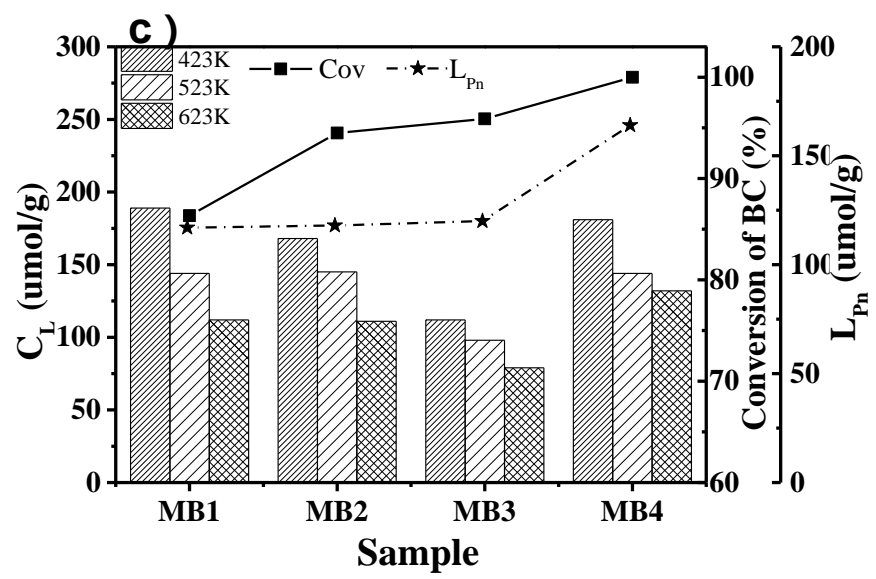

Figure 8. (a) The conversion of $\mathrm{BC}$ vs external surface area of the mesoporous Beta zeolites and the change with the accessibility of Brönsted acid sites. (b) Conversion of BC varies with the total acid amount and mesopore volume. (c) Conversion of BC varies with concentration of Lewis acid sites and their accessibility for mesoporous Beta zeolite MB1-4.

It is well known that the acid sites act as the active sites in the benzylation of naphthalene. The high concentration of acid sites in the catalyst should give a high conversion of the reactants. However, the results from our study did not fully agree with such a rule. As shown in Figure 8b, CB and MB1 did not give a high conversion of $B C$ even though they both had high concentrations of acid sites. Instead, the conversion of BC increased when the external surface area and mesoporosity increased. The reason for this phenomenon was that the accessibility of the acid sites played a significant role in the reaction. The high external surface area increased the accessibility of the active sites, which resulted in the high conversion of BC. Moreover, the increase of mesoporosity improved the diffusion of reactants and products, which enhanced the activity of the zeolite catalysts as well.

The concentration of Lewis acid sites at different temperatures reflected the strength of the acid sites. There is already research showing that Lewis acid is also important for benzylation of naphthalene [36]. The effect of Lewis acid on the conversion of BC was analyzed as well in this study. Figure 8c shows that MB1 and MB2 had a higher concentration of Lewis acid site than MB3 when the Py-IR results at different temperatures are compared. However, the concentration of Lewis acid sites from Pn-IR results showed that MB3 had better accessibility than MB1 and MB2. Consequently, as can be seen in Figure $8 c$, the conversion of $B C$ agreed with the trend of the accessibility of Lewis sites.

\section{Experimental}

\subsection{Catalyst Preparation}

Mesoporous Beta zeolites were synthesized by using the strategy we developed previously [14-17]. In practice, the mesoporous Beta zeolites were obtained by using organic functionalized fumed silica as the silicon source. The functionalization followed the same procedure as we developed [14]. Fumed silica was modified using TPOAC (dimethyloctadecyl-[3-(trimethoxysilyl) propyl] ammonium chloride) with different ratios of functionalization as described in Reference [17]. In a typical process, $30 \mathrm{~g}$ of fumed silica (Degussa, Aerosil 200, Marl, NRW, Germany) was dissolved in $600 \mathrm{~mL}$ of distilled water. The resultant mixture was then stirred at a rate of $100 \mathrm{rpm}$ under reflux at $373 \mathrm{~K}$ for $1 \mathrm{~h}$. After that, TPOAC (72 wt \% methanol solution, Aldrich, Saint Louis, MO, USA) was added into the former mixture and refluxed at $363 \mathrm{~K}$ for $10 \mathrm{~h}$. The product was washed several times with ethanol and dried at $373 \mathrm{~K}$ overnight.

The functionalized fumed silica was used as the silicon source for the synthesis of mesoporous Beta zeolite. A solution prepared by mixing distilled water, $\mathrm{NaOH}, \mathrm{NaAlO}_{2}$, and organic functionalized fumed silica, with a molar ratio of $30 \mathrm{SiO}_{2}: \mathrm{Al}_{2} \mathrm{O}_{3}: 7 \mathrm{Na}_{2} \mathrm{O}$, was stirred at room temperature for $4 \mathrm{~h}$, 
before being transferred into a teflon-coated stainless-steel autoclave. After being heated at $373 \mathrm{~K}$ for $12 \mathrm{~h}$, the solid product was washed with distilled water until the $\mathrm{pH}$ of the effluent was neutral, dried at $373 \mathrm{~K}$ overnight, and calcined in the presence of air at $873 \mathrm{~K}$ for $5 \mathrm{~h}$. The as synthesized mesoporous Beta was then ion exchanged three times using a solution of $1 \mathrm{M} \mathrm{NH}_{4} \mathrm{NO}_{3}$ with a liquid/solid ratio of $100 \mathrm{~mL} / \mathrm{g}$ at $333 \mathrm{~K}$ for $2 \mathrm{~h}$, followed by being washed with deionized water, dried at $393 \mathrm{~K}$ for $12 \mathrm{~h}$, and then calcined at $823 \mathrm{~K}$ for $3 \mathrm{~h}$. The resultant $\mathrm{H}$-mesoporous Beta zeolites were defined as MBn ( $\mathrm{n}=1-4$, following the increase of mesoporosity). A conventional microporous Beta was made for reference, following the same method but using non-functionalized fumed silica as the silicon source and named as $\mathrm{CB}$.

\subsection{Characterization}

The X-ray diffraction (XRD) measurements were carried out on a Shimadze XRD-6000 (Shimadzu, Nakagyo-ku, Kyoto, Japan) using $\mathrm{Cu} \mathrm{K} \alpha$ radiation in the $2 \theta$ range of $5^{\circ}-3^{\circ}$, with the step size and counting time of $0.02^{\circ}$ and $10 \mathrm{~s}$, respectively. Nitrogen adsorption-desorption isotherms were measured at $77 \mathrm{~K}$ on a Quantachrome QUADRASORB SI (Quantachrome Instruments, Boynton Beach, FL, USA). The samples were degassed at $613 \mathrm{~K}$ under vacuum for $3 \mathrm{~h}$ before the measurements. The total surface area was calculated using the BET equation, and the external surface area and micropore volume were obtained from the t-plot method. Pore size distribution was calculated using a non-local-density functional theory (NLDFT) method with a cylinder pore model in the Quantachrome's routines. Field emission scanning electron microscope (FESEM) images were obtained in a JEOL JSM-6700F (Jeol, Tokyo, Japan). ${ }^{29} \mathrm{Si}$ solid state magic angle spinning nuclear magnetic resonance (MAS-NMR) spectroscopy analysis was conducted on a Bruker Avance III $500 \mathrm{MHz}$ spectrometer (Bruker, Karlsruhe, Germany) operating at the resonance frequency of $99.36 \mathrm{MHz}$. The NMR spectra were fitted with Gaussian functions for the quantitative deconvolution of overlapping peaks.

Temperature-programmed desorption of ammonia ( $\mathrm{NH}_{3}$-TPD) was carried out in a TP-5076 TPD/TPR instrument (Xianquan, Tianjin, China) equipped with thermal conductivity detectors (TCD). About $100 \mathrm{mg}$ of the sample was granulated and sieved into particles $(200-400 \mu \mathrm{m})$ and activated at $823 \mathrm{~K}$ under the flow of argon for $1 \mathrm{~h}$. After cooling to $393 \mathrm{~K}$, the samples were saturated with $\mathrm{NH}_{3}$ for $1 \mathrm{~h}$ followed by degassing the samples for $30 \mathrm{~min}$ to remove the physically adsorbed $\mathrm{NH}_{3}$. After that, the temperature was increased to $873 \mathrm{~K}$ at a rate of $10 \mathrm{~K} / \mathrm{min}$, while desorption of $\mathrm{NH}_{3}$ was recorded. The Fourier transform infrared spectra (FTIR) of the Beta zeolite were measured on a Shimadzu IR Affinity-1 FTIR spectrometer (Shimadzu, Nakagyo-ku, Kyoto, Japan) at a resolution of $4 \mathrm{~cm}^{-1}$. The samples were prepared as self-supporting wafers $(\mathrm{d}=15 \mathrm{~mm})$ and activated in vacuum ( $\mathrm{p}=10^{-6} \mathrm{mbar}$ ) for $1 \mathrm{~h}$ at $673 \mathrm{~K}$ before the measurement. FTIR spectra of pyridine-adsorbed (Py-IR), 2,6-ditertbutylpyridine-adsorbed (DTBPy-IR), and pivalonitrile-adsorbed (Pn-IR) MBn were obtained on the same FTIR spectrometer. The samples were heated at $673 \mathrm{~K}$ under vacuum for $4 \mathrm{~h}$ before cooling to $423 \mathrm{~K}$. The samples were then exposed to pyridine $\left(\mathrm{p}=10^{-1} \mathrm{mbar}\right)$ for $1 \mathrm{~h}$ and then outgassed ( $p=10^{-6} \mathrm{mbar}$ ) at the measuring temperatures $(423 \mathrm{~K}, 523 \mathrm{~K}$, and $623 \mathrm{~K})$ for $1 \mathrm{~h}$ to remove the physically adsorbed pyridine, followed by the collection of the spectra. The concentration of total Brönsted and Lewis acid sites of the samples was calculated from peaks intensities at $1545 \mathrm{~cm}^{-1}\left(\mathrm{~B}_{\mathrm{Py}}\right)$ and at $1455 \mathrm{~cm}^{-1}\left(\mathrm{~L}_{\mathrm{Py}}\right)$ in the Py-IR spectra. For quantification, molar integral extinction coefficients of $2.22 \mathrm{~cm} / \mu \mathrm{mol}$ and $1.67 \mathrm{~cm} / \mu \mathrm{mol}$ were used for Brönsted and Lewis acid sites, respectively. The concentration of external Brönsted acid sites at $423 \mathrm{~K}$ was determined using the peak intensity at $1616 \mathrm{~cm}^{-1}\left(\mathrm{~B}_{\mathrm{DTBPy}}\right)$ in the DTBPy-IR spectra (molar integral extinction coefficient: $2.84 \mathrm{~cm} / \mu \mathrm{mol}$ ). The concentration of external Lewis acid sites measured by $\mathrm{Pn}$ at $303 \mathrm{~K}$ were calculated from peak intensities at $2294 \mathrm{~cm}^{-1}\left(\mathrm{~L}_{\mathrm{Pn}}\right)$ in the Pn-IR spectra (molar integral extinction coefficient: $\left.0.11 \mathrm{~cm} / \mu \mathrm{mol}\left(\mathrm{L}_{\mathrm{Pn}}\right)\right)$. 


\subsection{Catalytic Reaction Assessments}

The liquid-phase benzylation of naphthalene with benzyl chloride (BC) was conducted in a $50 \mathrm{~mL}$ three-necked flask equipped with a reflux condenser at $368 \mathrm{~K}$ with a nephathalene to $\mathrm{BC}$ molar ratio of 2:1. Before the reaction, the catalyst was activated at $723 \mathrm{~K}$ under an atmosphere of air overnight. The solution of naphthalene in cyclohexane with a volume of $30 \mathrm{~mL}$ was added to the system including the catalyst $(0.1 \mathrm{~g})$, and heated at $368 \mathrm{~K}$ for $30 \mathrm{~min}$, before $2 \mathrm{~mL}$ of BC was added. The mixture was continuously stirred at $368 \mathrm{~K}$ for $8 \mathrm{~h}$. After the catalyst was separated by centrifugation, the solution was withdrawn to be analyzed on a gas chromatograph that was equipped with an FID detector (Agilent, Santa Clara, CA, USA) and a $60 \mathrm{~m} \mathrm{HP-5}$ capillary column. Based on the consumption of benzyl chloride as the benzylating agent, and the amount of nephathalene was in excess, the conversion was calculated using the following equation:

$$
\text { Conversion }(\%)=\frac{w t \% \text { of consumed benzylchloride }}{w t \% \text { of initial benzylchloride }} \times 100 \%
$$

\section{Conclusions}

Introducing organic moiety into the zeolite synthesis system by using organic functionalized fumed silica as the silicon source significantly increased the mesoporosity and external surface area of the resultant mesoporous Beta zeolites. In addition, the mesoporosity and external surface area increased gradually when the content of organic moiety increased. The increased mesoporosity improved the accessibility of acid sites, which was demonstrated using an infrared spectroscopy study of adsorbed probe molecules including pyridine (Py-IR), 2,6-ditertbutylpyridine (DTBPy-IR), and pivalonitrile (Pn-IR). The mesoporous Beta zeolite catalysts showed enhanced activity in the benzylation of naphthalene with benzyl chloride, not only because of the increased accessibility of acid sites, but also because of the improved diffusion ability of the reactants and products. Such high performance of the mesoporous Beta zeolite catalysts was strongly influenced by the mesoporosity, acid concentration, acid type, acid strength, and most importantly the accessibility of the acid sites. With the increased mesoporosity and improved accessibility of acid sites, such mesoporous Beta zeolites could also give great performances in many other acid-catalyzed reactions involving bulky molecules.

Supplementary Materials: The following are available online at http:/ /www.mdpi.com/2073-4344/8/11/504/s1, Figure S1: 29Si MAS NMR spectra of mesoporous Beta zeolite MB1-3. Solid line is the original data; Dash dot data is the fitted data; Dash line is the cumulative fitted data. Figure S2: NH3-TPD for microporous Beta zeolite CB and mesoporous Beta zeolites MB1-4. Solid line is the original data; Dash dot line is the fitted data by Gaussian functions; Dash line is the cumulative fitted data. Figure S3: FTIR spectra of microporous Beta zeolite CB and mesoporous Beta zeolites MB1-3 after desorption of pyridine at three different temperatures. Figure S4: FTIR spectra of mesoporous Beta zeolites MB1-4 after desorption of DTBPy at 423K. Figure S5: FTIR spectra of mesoporous Beta zeolites MB1-4 after pivalonitrile (Pn) desorption at 303K. Table S1: Conversion of BA and Selectivity of products in the catalytic benzylation of naphthalene on microporous Beta zeolite CB and mesoporous Beta zeolites MB1-4

Author Contributions: W.H., J.M. and R.L. conceived and designed the experiments; W.Z. and Z.G. performed the experiments; W.H. and J.M. analyzed the data; R.L. contributed reagents/materials/analysis tools; W.H. wrote the paper.

Funding: This research was funded by the National Natural Science Foundation of China, grant number U1510127 and U1463209, and Shanxi Provincial Key Innovative Research Team in Science and Technology, grant number No. 2014131006.

Acknowledgments: This project was supported by the National Natural Science Foundation of China (Grant Nos. U1510127, U1463209) and Shanxi Provincial Key Innovative Research Team in Science and Technology (No. 2014131006).

Conflicts of Interest: The authors declare no conflict of interest. 


\section{References}

1. Higgins, J.B.; LaPierre, R.B.; Schlenker, J.L.; Rohrman, A.C.; Wood, J.D.; Kerr, G.T.; Rohrbaugh, W.J. The framework topology of zeolite beta. Zeolites 1988, 8, 446-452. [CrossRef]

2. Treacy, M.M.J.; Newsam, J.M. Two new three-dimensional twelve-ring zeolite frameworks of which zeolite beta is a disordered intergrowth. Nature 1988, 332, 249-251. [CrossRef]

3. Tanabe, K.; Hölderich, W.F. Industrial application of solid acid-base catalysts. Appl. Catal. A Gen. 1999, 181, 399-434. [CrossRef]

4. Degnan, T.F.; Smith, C.M.; Venkat, C.R. Alkylation of aromatics with ethylene and propylene: Recent developments in commercial processes. Appl. Catal. A Gen. 2001, 221, 283-294. [CrossRef]

5. Nivarthy, G.S.; He, Y.; Seshan, K.; Lercher, J.A. Elementary mechanistic steps and the influence of process variables in isobutane alkylation over H-BEA. J. Catal. 1998, 176, 192-203. [CrossRef]

6. Feller, A.; Guzman, A.; Zuazo, I.; Lercher, J.A. A novel process for solid acid catalyzed isobutane/butene alkylation. Sci. Technol. Catal. 2003, 145, 67-72.

7. Meng, X.; Nawaz, F.; Xiao, F.S. Templating route for synthesizing mesoporous zeolites with improved catalytic properties. Nano Today 2009, 4, 292-301. [CrossRef]

8. Valtchev, V.; Majano, G.; Mintova, S.; Pérez-Ramírez, J. Tailored crystalline microporous materials by post-synthesis modification. Chem. Soc. Rev. 2013, 42, 263-290. [CrossRef] [PubMed]

9. Li, K.; Valla, J.; García-Martínez, J. Realizing the Commercial Potential of Hierarchical Zeolites: New Opportunities in Catalytic Cracking. Chemctchem 2014, 6, 46-66. [CrossRef]

10. Hua, Z.L.; Zhou, J.; Shi, J.L. Recent advances in hierarchically structured zeolites: Synthesis and material performances. Chem. Commun. 2011, 47, 10536-10547. [CrossRef] [PubMed]

11. Serrano, D.P.; Escola, J.M.; Pizarro, P. Synthesis strategies in the search for hierarchical zeolites. Chem. Soc. Rev. 2013, 42, 4004-4035. [CrossRef] [PubMed]

12. Tao, Y.; Kanoh, H.; Abrams, L.; Kaneko, K. Mesopore-Modified Zeolites: Preparation, Characterization, and Applications. Chem. Rev. 2006, 106, 896-910. [CrossRef] [PubMed]

13. Na, K.; Choi, M.; Ryoo, R. Recent advances in the synthesis of hierarchically nanoporous zeolites. Microporous Mesoporous Mater. 2013, 166, 3-19. [CrossRef]

14. Xue, Z.; Ma, J.; Hao, W.; Bai, X.; Kang, Y.; Liu, J.; Li, R. Synthesis and characterization of ordered mesoporous zeolite LTA with high ion exchange ability. J. Mater. Chem. 2012, 22, 2532-2538. [CrossRef]

15. Dai, G.; Ma, J.; Xiao, H.; Hao, W.; Li, R. Mesoporous EU-1 zeolite with enhanced accessibility and diffusion for bulky molecular reaction. Mater. Res. Bull. 2018, 100, 76-82. [CrossRef]

16. Dai, G.; Hao, W.; Xiao, H.; Ma, J.; Li, R. Hierarchical mordenite zeolite nano-rods bundles favourable to bulky molecules. Chem. Phys. Lett. 2017, 686, 111-115. [CrossRef]

17. Zhang, Q.; Ming, W.; Ma, J.; Zhang, J.; Wang, P.; Li, R. De novo assembly of a mesoporous beta zeolite with intracrystalline channels and its catalytic performance for biodiesel production. J. Mater. Chem. A 2014, 2, 8712-8718. [CrossRef]

18. Coq, B.; Gourves, V.; Figuéras, F. Benzylation of toluene by benzyl chloride over protonic zeolites. Appl. Catal. A Gen. 1993, 100, 69-75. [CrossRef]

19. Choudhary, V.R.; Jana, S.K.; Kiran, B.P. Alkylation of benzene by benzyl chloride over H-ZSM-5 zeolite with its framework Al completely or partially substituted by Fe or Ga. Catal. Lett. 1999, 59, 217-219. [CrossRef]

20. Kim, J.C.; Cho, K.; Lee, S.; Ryoo, R. Mesopore wall-catalyzed Friedel-Crafts acylation of bulky aromatic compounds in MFI zeolite nanosponge. Catal. Today 2015, 243, 103-108. [CrossRef]

21. Kim, J.C.; Cho, K.; Ryoo, R. High catalytic performance of surfactant-directed nanocrystalline zeolites for liquid-phase Friedel-Crafts alkylation of benzene due to external surfaces. Appl. Catal. A Gen. 2014, 470, 420-426. [CrossRef]

22. Jin, H.; Ansari, M.B.; Jeong, E.Y.; Park, S.E. Effect of mesoporosity on selective benzylation of aromatics with benzyl alcohol over mesoporous ZSM-5. J. Catal. 2012, 291, 55-62. [CrossRef]

23. Yutthalekha, T.; Wattanakit, C.; Warakulwit, C.; Wannapakdee, W.; Rodponthukwaji, K.; Witoon, T.; Limtrakul, J. Hierarchical FAU-type zeolite nanosheets as green and sustainable catalysts for benzylation of toluene. J. Clean. Prod. 2017, 142, 1244-1251. [CrossRef]

24. Miao, H.; Zhang, W.; Hu, S.; Ma, J.; Li, R. A practicable mesostructured MFI zeolitic catalyst for large molecule reactions. Catal. Commun. 2016, 78, 68-70. [CrossRef] 
25. Leng, K.; Wang, Y.; Hou, C.; Lancelot, C.; Lamonier, C.; Rives, A.; Sun, Y. Enhancement of catalytic performance in the benzylation of benzene with benzyl alcohol over hierarchical mordenite. J. Catal. 2013, 306, 100-108. [CrossRef]

26. Sing, K.S.W.; Everett, D.H.; Haul, R.A.W.; Moscou, L.; Pierotti, R.A.; Rouquerol, J. Reporting physisorption data for gas/solid systems with special reference to the determination of surface area and porosity. Pure Appl. Chem. 1985, 57, 603-619. [CrossRef]

27. Simon-Masseron, A.; Marques, J.P.; Lopes, J.M.; Ribeiro, F.R.; Gener, I.; Guisnet, M. Influence of the Si/Al ratio and crystal size on the acidity and activity of HBEA zeolites. Appl. Catal. A Gen. 2007, 316, 75-82. [CrossRef]

28. Maier, S.M.; Jentys, A.; Lercher, J.A. Steaming of zeolite BEA and its effect on acidity: A comparative NMR and IR spectroscopic study. J. Phys. Chem. C 2011, 115, 8005-8013. [CrossRef]

29. Busca, G. Acidity and basicity of zeolites: A fundamental approach. Microporous Mesoporous Mater. 2017, 254, 3-16. [CrossRef]

30. Sandoval-Díaz, L.-E.; Gonzalez-Amaya, J.-A.; Trujillo, C.-A. General aspects of zeolite acidity characterization. Microporous Mesoporous Mater. 2015, 215, 229-243. [CrossRef]

31. Candu, N.; Florea, M.; Coman, S.M.; Parvulescu, V.I. Benzylation of benzene with benzyl alcohol on zeolite catalysts. Appl. Catal. A Gen. 2011, 393, 206-214. [CrossRef]

32. Parry, E.P. An Infrared Study of Pyridine Adsorbed Characterization of Surface. J. Catal. 1963, 379, $371-379$. [CrossRef]

33. Koo, J.B.; Jiang, N.; Saravanamurugan, S.; Bejblová, M.; Musilová, Z.; Čejka, J.; Park, S.E. Direct synthesis of carbon-templating mesoporous ZSM-5 using microwave heating. J. Catal. 2010, 276, 327-334. [CrossRef]

34. Trombetta, M.; Busca, G.; Lenarda, M.; Storaro, L.; Pavan, M. An investigation of the surface acidity of mesoporous Al-containing MCM-41 and of the external surface of ferrierite through pivalonitrile adsorption. Appl. Catal. A Gen. 1999, 182, 225-235. [CrossRef]

35. Kuehl, G.H.; Timken, H.K.C. Acid sites in zeolite Beta: Effects of ammonium exchange and steaming. Microporous Mesoporous Mater. 2000, 35, 521-532. [CrossRef]

36. Bejblová, M.; Procházková, D.; Čejka, J. Acylation reactions over zeolites and mesoporous catalysts. ChemSusChem 2009, 2, 486-499. [CrossRef] [PubMed] 